\title{
A mobile insulator system to detect and disrupt cis-regulatory landscapes in vertebrates
}

\author{
José Bessa, ${ }^{1,3}$ Mario Luengo, ${ }^{2}$ Solangel Rivero-Gil, ${ }^{2}$ Ana Ariza-Cosano, ${ }^{2}$ \\ António H.F. Maia, Francisco J. Ruiz-Ruano, Pablo Caballero, Silvia Naranjo, \\ Jaime J. Carvajal, and José Luis Gómez-Skarmeta ${ }^{3}$ \\ Centro Andaluz de Biología del Desarrollo (CABD), CSIC-Universidad Pablo de Olavide-Junta de Andalucía, Seville 41013, Spain
}

\begin{abstract}
In multicellular organisms, cis-regulation controls gene expression in space and time. Despite the essential implication of cisregulation in the development and evolution of organisms and in human diseases, our knowledge about regulatory sequences largely derives from analyzing their activity individually and outside their genomic context. Indeed, the contribution of these sequences to the expression of their target genes in their genomic context is still largely unknown. Here we present a novel genetic screen designed to visualize and interrupt gene regulatory landscapes in vertebrates. In this screen, based on the random insertion of an engineered Tol2 transposon carrying a strong insulator separating two fluorescent reporter genes, we isolated hundreds of zebrafish lines containing insertions that disrupt the cis-regulation of tissue-specific expressed genes. We therefore provide a new easy-to-handle tool that will help to disrupt and chart the regulatory activity spread through the vast noncoding regions of the vertebrate genome.
\end{abstract}

[Supplemental material is available for this article.]

Transcriptional regulation is achieved by a multitude of cis-regulatory elements (CREs) that control spatiotemporal gene activation and expression levels. Genes with complex expression patterns are regulated by multiple CREs spread over large genomic distances that together define regulatory landscapes (Spitz et al. 2003; Ragvin et al. 2010). The borders of these landscapes are often flanked by insulators, a specific type of CREs that prevent spurious interactions between adjacent regulatory landscapes (Geyer and Corces 1992; Chung et al. 1993; Bell et al. 2001; Martin et al. 2011; Dixon et al. 2012). Moreover, disruption of these landscapes is at the basis of many human genetic diseases, which can occur by different mechanisms ranging from large chromosomal aberrations, such as translocations, inversions, deletions, or duplications, to more subtle modifications like point mutations in particular CREs (Epstein 2009; Kleinjan and Coutinho 2009; Kilpinen and Dermitzakis 2012). Since these diseases are caused by improper gene regulation, rather than by a complete loss of gene function, it becomes imperative to systematically isolate this kind of regulatory perturbations in animal models. Although the use of next-generation sequencing techniques coupled to in vivo reporter assays has enormously facilitated the identification of CREs (Visel et al. 2009; Blow et al. 2010), there is still little information on the actual contribution of these noncoding regions to gene expression in their genomic context, how they are organized in regulatory landscapes, and the functional consequences of their disruption (Lettice et al. 2002; Jeong et al. 2008; Visel et al. 2010; Ghiasvand et al. 2011; Collette et al. 2012). To fill this gap and to generate a tool that permits an unbiased genomic approach, we have designed an expression disruption (ED) system based on the Tol2

\footnotetext{
'Present address: Instituto de Biologia Molecular e Celular (IBMC), 4150-180 Porto, Portugal

transposon that harbors a strong insulator. This approach allows for the disruption and detection in vivo of complex regulatory landscapes and constitutes the first screen in vertebrates specifically designed to impair enhancer-promoter interaction. We have isolated 223 zebrafish ED lines that can be visualized through the EDscreen browser (http://www.upo.es/CABD/EDscreen/). A detailed evaluation of a fraction of the ED lines shows that in at least half of them, the cis-regulatory landscapes have been disrupted, causing alterations on gene expression and function. In vivo deletion of the ED insulator together with chromosome conformation capture (3C) (Dekker et al. 2002) assays demonstrate that the disruption of regulatory landscapes depends on the insulator, which impairs proper enhancer/promoter interactions. Finally, we also present preliminary evidence indicating that our system might be directly implemented in mouse embryos. Collectively, our data demonstrate that ED is an easy to use tool that can be applied in a variety of animal models to induce regulatory mutations caused by tissue-specific alterations on gene expression.

\section{Results}

ED is an engineered Tol2 transposon (Kawakami et al. 2000) that includes a strong insulator flanked by two fluorescent reporter genes under the control of minimal promoters that function as independent enhancer traps (Fig. 1). The strong insulator in the ED transposon is expected to disrupt the regulatory landscapes in which it becomes integrated. Therefore, the regulatory information upstream of or downstream from the integration site should differentially activate green fluorescent protein (GFP) or red fluorescent protein (RFP) reporters, positioned on either side of the insulator (Fig. 1). Out of $224 \mathrm{ED}$ injected embryos grown to adults, we

\footnotetext{
(C) 2014 Bessa et al. This article is distributed exclusively by Cold Spring Harbor Laboratory Press for the first six months after the full-issue publication date (see http://genome.cshlp.org/site/misc/terms.xhtml). After six months, it is available under a Creative Commons License (Attribution-NonCommercial 3.0 Unported), as described at http://creativecommons.org/licenses/by-nc/3.0/.
} 


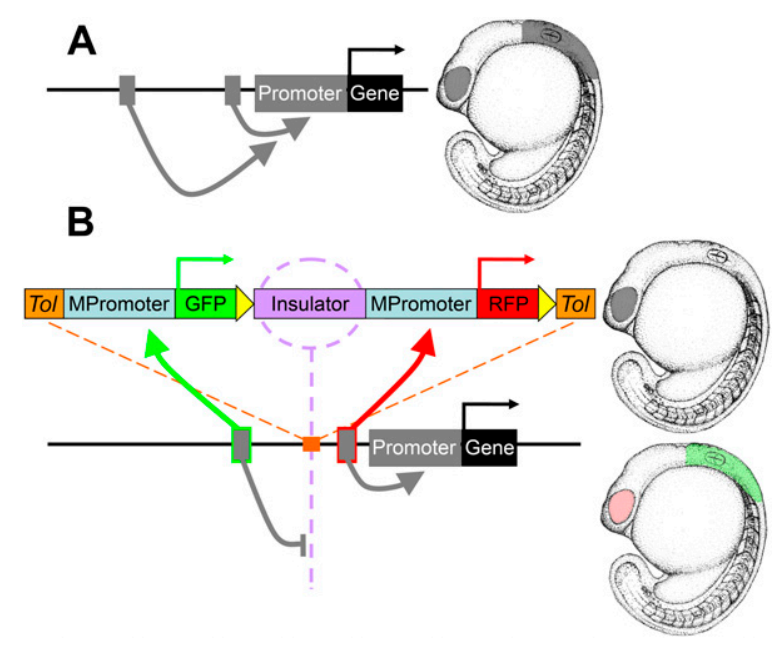

Figure 1. The expression disruption system. $(A)$ Representation of a genomic landscape composed of two enhancers (gray boxes) that drive expression of their target gene in the eye and hindbrain (gray pattern in embryo). (B) An ED insertion in this genomic landscape. ED is composed of two enhancer traps, one that has GFP (green box) as a reporter gene and the other RFP (red box). An insulator is present in between these two enhancer traps (purple box). Two loxP sequences (yellow triangles) flank the RFP enhancer trap and the insulator. In this insertion, the upstream enhancer is detected exclusively by the GFP enhancer trap cassette, and the downstream is detected only by the RFP cassette, resulting in the nonoverlapping expression of these two reporters (green and red patterns in the lower embryo). The block of the upstream enhancer by the insulator results in a regulatory mutation (higher embryo).

isolated 223 stable transgenic lines and annotated GFP and RFP expression patterns at 24 and $48 \mathrm{~h}$ post-fertilization (hpf) (Supplemental Table 1). The vast majority of these lines show different expression patterns for the green and red fluorescent proteins $(n=$ 173, 78\%) (Fig. 2A-C), comprising cases of GFP and RFP expression in different tissues ( $n=143)$ (Fig. $2 \mathrm{~A})$ and cases of GFP $(n=18)$ or RFP expression alone ( $n=12$ ) (Fig. $2 \mathrm{~B}, \mathrm{C})$. These results suggest that both enhancer traps are detecting different genomic areas of the original regulatory landscape that has been separated by the insulator (Fig. 1). A fraction of lines ( $n=50,22 \%)$ (Fig. 2D) showed total coexpression of GFP and RFP, suggesting redundancy of regulatory elements upstream of and downstream from the ED integration site or the failure of the insulator activity in these genomic regions. Southern blot analysis of a fraction of the generated ED lines (19 lines analyzed) indicated that in the majority of them (74\%), the GFP and RFP expression patterns are caused by single insertions (Supplemental Fig. 1). To determine if the ED vector has a tissuespecific bias, we compared the percentage of ED lines and genes that shows expression in a given tissue and observed that the distribution of both groups is largely similar (Supplemental Fig. 2).

To test that the segregation of the GFP and RFP expression patterns are due to the insulator activity, we included in the ED's design two loxP sequences flanking the insulator and the RFP trap cassette, which allows for the excision of this sequence by the activity of Cre recombinase (Fig. 1B). By injecting Cre mRNA into eight ED lines that show little overlap of RFP/GFP expression, we consistently observed the loss of RFP with the concomitant gain of GFP expression in territories where RFP was originally expressed (Supplemental Figs. 3, 4). This indicates that the excision cassette was effectively removed in vivo, which allowed the GFP reporter to read the regulatory information previously acting on the RFP gene.
We therefore conclude that the built-in ED insulator can efficiently segregate regulatory landscapes.

To determine if ED is able to recapitulate the expression pattern of genes near the integration site, first we have mapped $59 \mathrm{ED}$ lines by inverse PCR (Supplemental Fig. 5; Supplemental Table 2). Next, we compared the expression pattern of these lines with those from the genes near the insertions, being able to associate an ED line to a likely target gene in 32 cases (Supplemental Fig. 6). Three of such cases-ED25, ED27, and ED170-were linked to klf4b, dacha, and ptrfb, respectively (Fig. 3A-C, first and second columns), in which reporters and associated genes show remarkably similar expression patterns. Another interesting example is the line ED186, which was mapped to $0.9 \mathrm{~kb}$ upstream of mir124-5. This line shows a strong expression of RFP in the central nervous system (CNS) and eye (Fig. 3D), coinciding with the expression of this microRNA (Wienholds et al. 2005) and contrasting with a much-restricted GFP expression. Previous studies have reported that a fragment of $5.6 \mathrm{~kb}$ immediately upstream of mir124-5 is sufficient to recapitulate the expression pattern of this microRNA (Shkumatava et al. 2009), suggesting that most of its CREs are contained within this genomic region. The ED186 insertion separates this fragment into two 4.7-kb and 0.9-kb regions flanking RFP and GFP, respectively (Fig. 3E). Therefore, our insertion predicts that mir124-5 CREs are mainly located within the $4.7-\mathrm{kb}$ genomic region. This was confirmed by enhancer assays using the $4.7-\mathrm{kb}$ and $0.9-\mathrm{kb}$ fragments, which showed that the first region was able to drive an expression pattern largely similar to the mir124 and ED186 (Fig. 3F), while the second one was not able to drive transgene expression (Supplemental Fig. 7). These results demonstrate that ED insertions recapitulate the regulatory landscape of nearby genes and validate the ED's potential to predict the position of CREs in the genome.

To further examine if the disruption of regulatory landscapes caused by ED insertions affects endogenous gene expression, we selected eight of the 32 lines that were associated to a particular gene and performed in-crosses to generate homozygous embryos for these insertions. For seven of these ED lines, we performed in situ hybridizations for the transcript of the associated genes. Embryos homozygous for ED25, ED27, and ED170 show clear tissuespecific down-regulation of their respective target genes, $k l f 4 b$, dacha, and ptrfb (Fig. 3A-C, third column). A similar result was obtained for the ED186 line, which shows decreased levels of mir124-5, when assessed by qPCR (Fig. 3G). As these insertions do not overlap with the coding sequences of the associated genes (Supplemental Table 2), the down-regulation of their associated gene must be caused by cis-regulatory mutations that disrupt tissue-specific gene expression.

To demonstrate that the observed alteration of gene expression is induced by the insulator in the ED vector and not simply by the insertion of Tol2, we excised the insulator by injecting Cre recombinase mRNA in the $k l f 4 b$, dacha, and ptrfb ED-mutant backgrounds. In all cases, we observed an almost complete rescue of the transcript levels (Fig. 3A-C, fourth column; Supplemental Fig. 8). To better understand the impact of ED insertions on regulatory landscapes, we selected the line ED170, which carries an insertion near the gene ptrfb. Using epigenetic data that we recently generated in zebrafish (Bogdanovic et al. 2012), we searched for potential enhancers that could regulate the expression of this gene (Fig. 4A; Supplemental Fig. 9). Three of four tested sequences were able to drive expression of GFP in the notochord, recapitulating the expression pattern of ptrfb and ED170 (Fig. 4B). To activate gene expression, enhancers need to contact their target promoters physically. These interactions are mediated by long-range chromatin

\section{Genome Research}




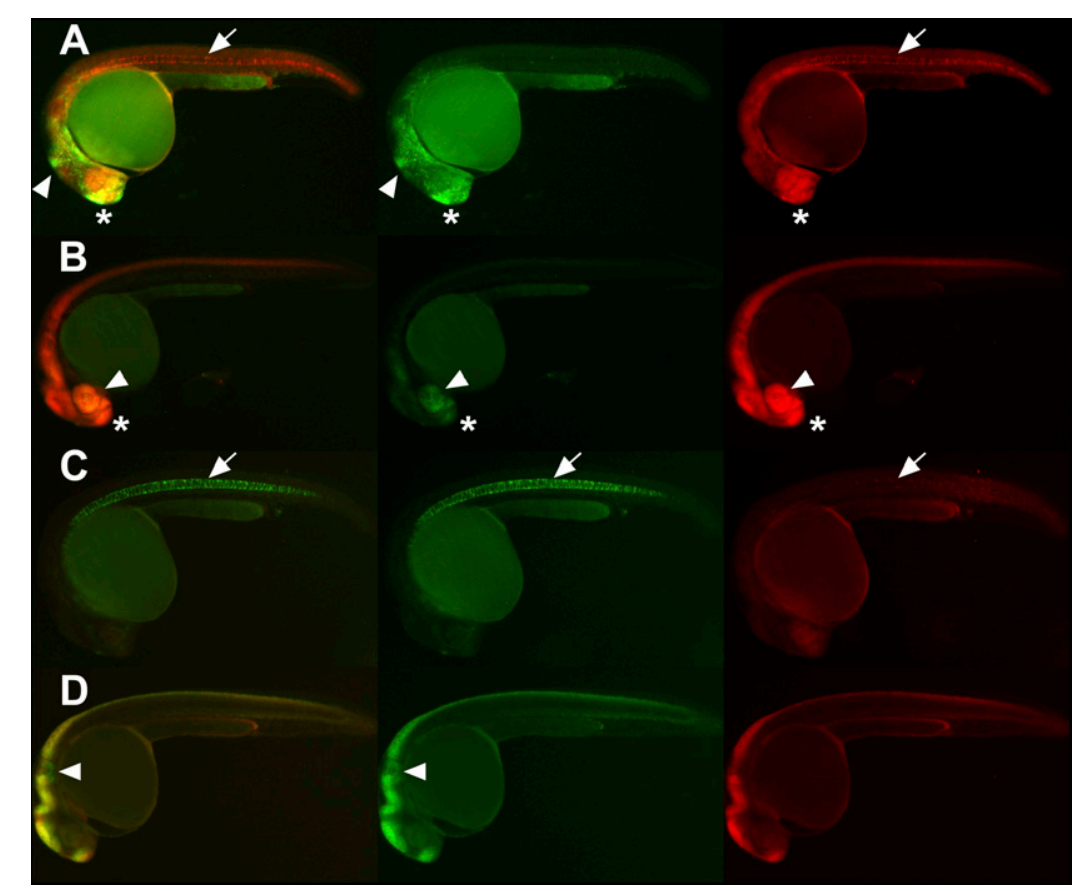

Figure 2. Expression disruption lines. $(A-C)$ The lines ED185b $(A)$, ED186 (B), and ED170 (C) show separated expression of GFP (green) and RFP (red). ED185b has expression of GFP in the neural crest (arrowhead) and RFP in the notochord (arrow). Also, both reporters show coexpression in the forebrain (asterisk). ED186 shows predominant expression of RFP in the eye (arrowhead) and central nervous system, while expression of GFP is restricted to very mild levels in the eye (arrowhead) and forebrain (asterisk). ED170 shows predominant expression of GFP in the notochord (arrow). (D) ED162 shows mostly coexpression of GFP and RFP in the central nervous system, having an exclusive domain of GFP expression in the otic vesicle (arrowhead).

loops that can be detected by the chromosome conformation capture (3C) technique (Dekker et al. 2002). Performing 3C assays in wild-type embryos, we showed a robust interaction of the most distal enhancer with the ptrfb promoter (enhancer 1) (Fig. 4C). Interestingly, this interaction was impaired when the 3C assay was performed in embryos homozygous for the ED170 insertion (Fig. 4C). These results correlate with a decrease of ptrfb transcription in the notochord, which might indicate that the interaction of the identified distal enhancer with the prtfb promoter is required for proper $p t r f b$ notochord expression. In summary, these results suggest that the ED system disrupts cis-regulatory landscapes by preventing enhancer-promoter interactions.

We next explored the phenotypes associated with ED lines. To that end, we focus on ED170 and ED25, two mutant lines that show a reduction of transcription of $p t r f b$ and $k l f 4 b$, respectively. In the case of ED170, 50\% of the homozygous embryos show some indentations in the notochord (Fig. 5A), a phenotype that was almost completely rescued after injection of Cre recombinase mRNA (Fig. 5B). When analyzing these indentations by confocal microscopy, nuclei are detected in a nuclei-free region of the notochord (Fig. 5C), suggesting an important disorganization of the notochord tissue. As expected, this very mild phenotype can be mimicked by injecting low amounts of a morpholino that specifically targets ptrfb (3 ng; Fig. 5D). The injection of higher amounts of this morpholino (10 ng) generated a much stronger phenotype that consisted of highly bent embryos, as previously reported (Supplemental Fig. 10; Hill et al. 2008). This indicates that homozygous ED170 insertion causes a partial knockdown of this gene. The other insertion, ED25, showed no detectable phenotype in homozygosis, despite its strong disruption of $k l f 4 b$ expression in the blood island. This gene has been shown to be required for the expression of hemoglobin beta embryonic-3 (previously beta E3 globin) (Gardiner et al. 2007). Therefore, we examined hemoglobin beta embryonic-3 expression in homozygous ED25 embryos and found a clear decrease in the transcription levels of this gene (Supplemental Fig. 10). This indicates that although there are no clear morphological defects in ED25 homozygous embryos, there are some clear effects in trans associated to this insertion, which are detectable using molecular markers.

Finally, we also wanted to know if the newly developed ED system could be used in other vertebrate models. Insulators are common CREs present in all vertebrate genomes, and although the ED's insulator is a chimera of chicken (Chung et al. 1993) and mouse (Giraldo et al. 2003) insulators, it is functional in zebrafish, evidencing that these elements function similarly in many different vertebrate genomes. As it has been shown that Tol2 is also effective in mouse transgenesis (Sumiyama et al. 2010), we injected the ED vector along with the Tol 2 mRNA in mouse embryos at the 1-cell stage as reported (Sumiyama et al. 2010). The injected embryos were then transferred into pseudopregnant females and isolated at 10.5-11.5 d post-coitum (dpc). From a total of 94 embryos, eight showed clear GFP and/or RFP expression, in several cases in different tissues (Fig. 6). These results suggest that the ED system could be implemented in a mammal model such as the mouse.

\section{Discussion}

We have developed the ED vector system as a new tool to interfere with the interaction between enhancers and promoters, which results from the random introduction of a strong insulator in the genome by means of the Tol2 transposon. In our system, the presence of this strong insulator within the regulatory landscape of a given gene results in a partial disruption of the transcriptional environment of the locus, thus having the potential to generate new phenotypes derived from the improper regulation of the target gene. As the ED vector carries two different reporter genes, located at either side of the insulator, it allows for the pre-selection in $\mathrm{F}_{1}$ zebrafish embryos, of those insertions with stronger disruptive activity as determined by simple visual inspection of the reporter genes' expression patterns, thus potentially making it very efficient for the screening of new regulatory mutations. Furthermore, preliminary data of the use of this system in mouse embryos indicate that it may be amenable for its use in large-scale regulatory screens in other vertebrates.

In this study, we establish a proof-of-principle for a method to disrupt enhancer-promoter interactions. Furthermore, we describe in detail an ED insertion in the ptrfb locus that impairs the interaction of at least one enhancer with its cognate promoter, resulting 


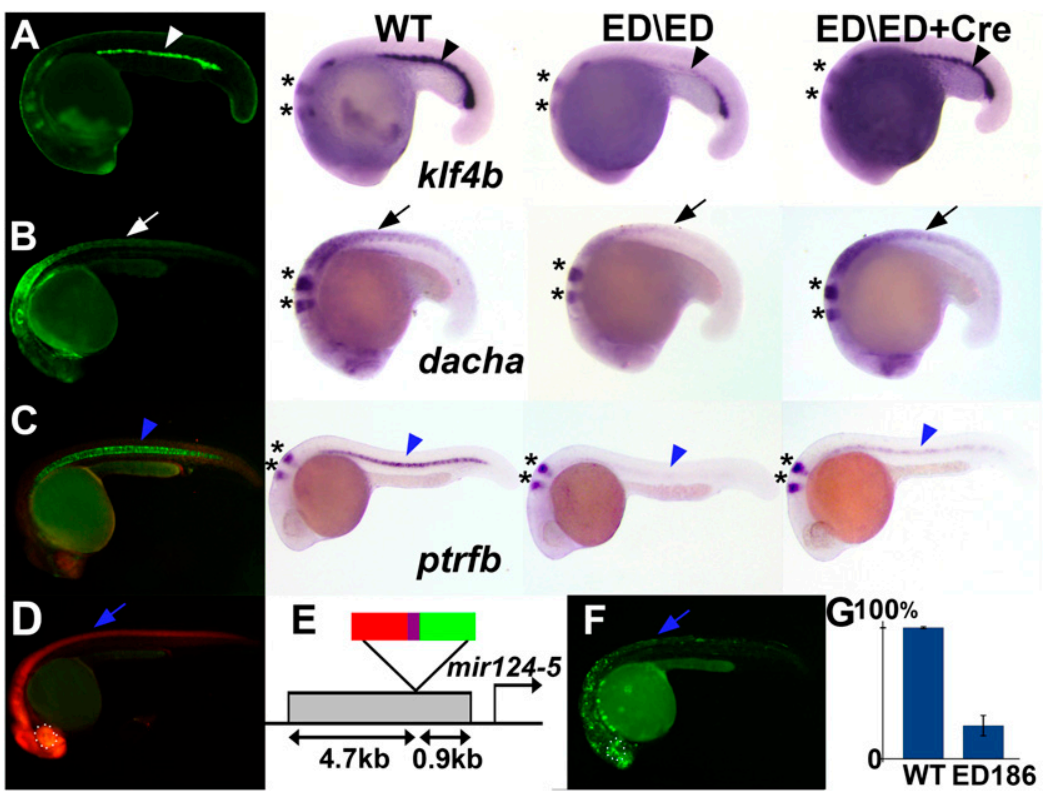

Figure 3. Example of insertions that cause mutations in nearby associated genes. (A) ED25 GFP expression recapitulates the expression pattern of $k$ lf $4 b$ detected by in situ hybridization, in the blood island (white and black arrowheads). (B) ED27 shows expression of GFP in the spinal cord (white arrow), which coincides with the expression of dacha (black arrow). (C) ED170 shows a strong expression of GFP in the notochord (blue arrowhead), recapitulating the expression of $p t r f b$ (blue arrowhead). ( $A-C$ ) In all three cases, homozygous embryos for the insertions show decreased levels of transcripts for the associated genes (third column), and the endogenous expression is recovered when Cre recombinase is injected in this homozygous mutant background (fourth column). Asterisks mark the expression of egr $2 b$, a marker used as an internal control for the in situ hybridization. (D) ED186 shows strong RFP expression in the central nervous system (blue arrow) and eye (white dotted circle). ( $E$ ) This line is an ED insertion near mir1 24-5 oriented with the RFP enhancer trap upstream of this noncoding gene. $(F)$ In ED186, an assay for enhancer activity of the sequence $4.7 \mathrm{~kb}$ upstream of the insertion point reveals expression in the central nervous system (blue arrow) and eye (white dotted circle). (G) Homozygous embryos for ED186 insertion show a decrease of $>70 \%$ in the transcription levels of mir $124-5$, detected by qPCR.

in a decrease on gene transcription levels. Strikingly, the phenotypes associated with this insertion differ from those obtained by a strong hypomorphic condition generated by standard amounts of morpholinos targeting ptrfb (Hill et al. 2008; this study). In the case of our regulatory disruption, the phenotypes are very mild, affecting only discrete notochord sections where abnormal indentations are observed, while the use of morpholinos gives rise to generalized notochord malformation resulting in a bent tail phenotype. These results highlight that quantitative differences in the activity of genes might result in important, and potentially difficult to predict, qualitative differences in phenotype. Therefore, the study of regulatory mutations should be essential to understand the biological function of regulatory sequences. In addition, and from a biomedical perspective, animal models harboring regulatory mutations might help to understand the etiology of human diseases associated to this class of mutations.

We found that in the majority of the insertions generated (78\%), the green and red fluorescent proteins are differentially expressed, suggesting that the insulator incorporated into the ED vector efficiently interferes in vivo with enhancerpromoter interactions. The ED insulator is composed of two sequences, the chicken HS4 insulator (Chung et al. 1993) and a sequence from the LCR located upstream of the mouse Tyr gene (Giraldo et al. 2003). By using an insulator assay in zebrafish, we show that the two sequences, which independently have insulator activity (Bessa et al. 2009), are much more effective insulators when linked together. CTCF, a DNA-binding protein largely associated with insulator activity (Phillips-Cremins and Corces 2013), binds to the chicken HS4 insulator, but according to previously published available data (The Mouse ENCODE Consortium 2012) does not appear to be associated to the Tyr insulator. Analysis of the available data on TFIIIC binding (The ENCODE Project Consortium 2012), another insulatorassociated protein (Kirkland et al. 2013), seems to indicate that this protein does not bind the Tyr insulator either. Therefore, there is the possibility that the Tyr insulator may operate through a CTCF/ TFIIIC-independent mechanism. This could explain the high efficiency of the ED insulator when compared with the work by Sanyal and colleagues, which shows that the majority of enhancer/ promoter long-range interactions (79\%) detected by chromosome conformation capture carbon copy (5C) are not blocked by the presence of one or more CTCF-binding sites detected by ChIP-seq (Sanyal et al. 2012).

We conclude that ED is a highly efficient tool, simple to use, that can significantly help to fill an important gap in the study of

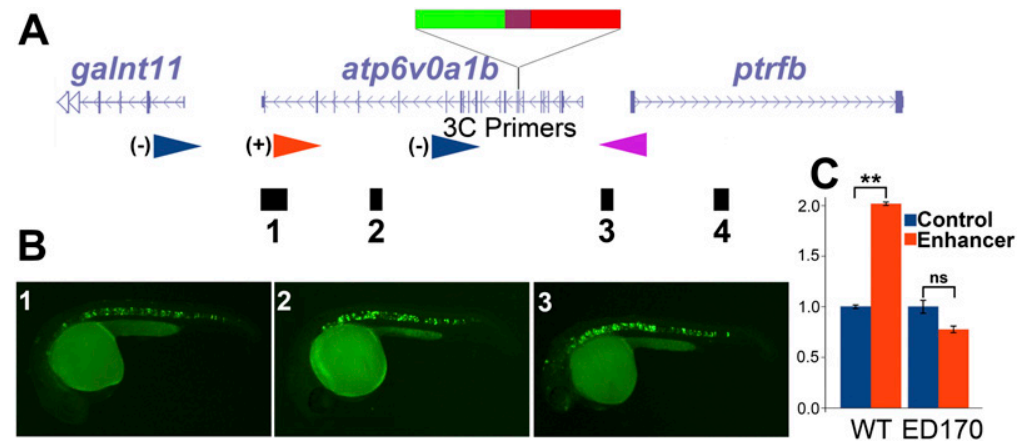

Figure 4. The genomic landscape of the ED170 insertion. (A) ED170 is an insertion in an exon of atp6v0a1b, $10 \mathrm{~kb}$ away from the transcription start site of its associated gene, ptrfb. This insertion is oriented with the GFP enhancer trap upstream of ptrfb. Four candidate enhancer sequences were selected, 1 to 4 (black boxes). (B) Enhancer activity assays show that sequences 1, 2, and 3 are enhancers that drive expression in the notochord, as observed by expression of GFP. (C) When performing a $3 C$ assay comparing the levels of interaction of the enhancer 1 and two control sequences with the promoter of $p t r f b$, a significant difference is observed; $\left({ }^{*}\right) P<0.01$. This same assay was performed using homozygous embryos for ED170, and the difference in the levels of interaction of the enhancer 1 and the two control sequences is not statistically significant (ns, not significant). The primers used for the $3 C$ experiment are represented in $A$ as an orange triangle for the enhancer 1, blue triangles for control sequences, and a magenta triangle for the ptrfb promoter sequence.

\section{Genome Research www.genome.org}




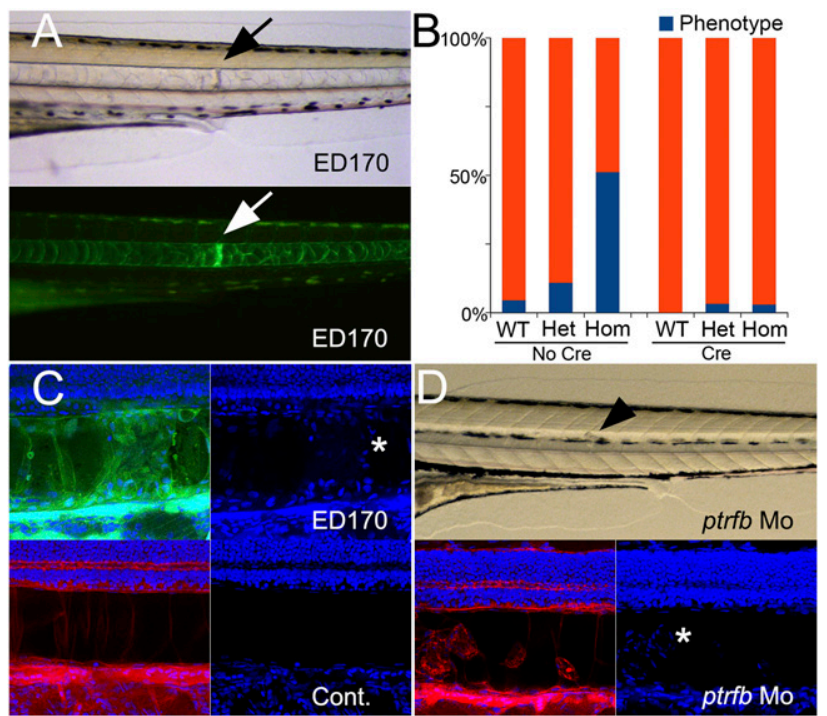

Figure 5. Phenotype associated with the ptrfb/ED170 mutant line. $(A)$ In homologous ptrfb/ED170 mutant embryos, indentations in the notochord are observed (arrows). Transmitted light (top) and GFP fluorescence (bottom) images are presented. (B) Graph representing the percentage of embryos, wild-type (WT), heterozygous (Het), or homozygous (Hom) for the ED170 insertion, presenting the described phenotype (blue) in uninjected (No Cre; $n=354$ ) and in Cre mRNA-injected backgrounds (Cre; $n=$ 126). (C) Indentations in the ED170 mutant background (ED170; top panels) correspond to nuclei-enriched regions in the notochord (asterisk) that in controls remain nuclei-free (Cont.; lower panels). (D) In morpholinoinjected embryos targeting ptrfb, indentations (black arrowhead) that correspond to nuclei-enriched regions in the notochord (asterisk) are also detected. Confocal images are marked in green for GFP, red for rodaminphalloidin/actin staining, and blue for DAPI/nuclei staining.

the contribution of the noncoding DNA to gene expression and function. Classical reverse genetics, which is usually phenotypedriven (Driever et al. 1996; Haffter et al. 1996), is difficult to use due to the mild phenotypes that might be expected from hypomorphic mutations obtained by the disruption of regulatory landscapes. A clear example of these mild phenotypes, hard to detect by simple phenotyping, is shown by some of the mutants isolated in this screen. More recent mutagenic methods that generate small changes in specific sequences, as high-throughput TILLIG (targeting induced local lesions in genomes) (Wienholds et al. 2003) or site-directed mutagenesis (Doyon et al. 2008; Meng et al. 2008; Bedell et al. 2012; Hwang et al. 2013), are highly efficient for coding sequences, but they are hard to apply to the noncoding genome, mostly because of our limited ability to predict the outcome of small changes in noncoding sequences. Classical random insertional mutagenesis might be used to disrupt single enhancers by direct hits (Golling et al. 2002), a potential that it is still present in our ED system. Recently, several methods have been developed to introduce loxP sequences into the genome (Ruf et al. 2011; Bedell et al. 2012; Chang et al. 2013), offering the potential to delete CREs. Because ED carries loxP sites, it might be used together with these loxP-based strategies to generate site-specific deletions, giving form to a much more powerful system with the ability to detect, locate, disrupt, and delete CREs. Our preliminary results in mouse embryos indicate that our ED system can likely be used to disrupt regulatory landscapes in multiple animal models. This will become very useful in the near future in light of recent findings that have shown that most of the genetic variants associated with human diseases identified by genome wide association studies (GWAS) lie also in non- coding sequences with likely cis-regulatory activity (Maurano et al. 2012).

\section{Methods}

\section{ED constructs}

Four different constructs were built and used to perform the ED screen: IMP16, IMP17, IMPCherry, and IIC. All of them contain a GFP transgene whose expression is controlled by a gata $2 a$ minimal promoter (Bessa et al. 2009), a fusion of the GAB insulator from the mouse tyrosinase gene (Giraldo et al. 2003), and the 5'HS4 insulator from the chicken beta-globin gene (Chung et al. 1993; Recillas-Targa et al. 2002) and the mini-Tol2 backbone (Urasaki et al. 2006). IMP16 and IMP17 contain in the RFP enhancer trap, the meis1 minimal promoter driving the DsRed 2 reporter gene. These two constructs differ in the orientation of the gata2a-GFP enhancer trap. In IMP16, the configuration is meis1-DsRed2-INSULATORS-gata2a-GFP, and in IMP17 is meis1-DsRed2-INSULATORS-GFP-gata2a. IMPCherry is similar to IMP17 but with the mCherry transgene (Kwan et al. 2007) instead of DsRed2. In IMP16, IMP17, and IMPCherry constructs, a myc-tag N-terminal fusion to RFP was engineered (Supplemental Fig. 11). These three constructs were used to generate ED1-ED47 transgenic lines (Supplemental Table 1). These lines show very weak or undetectable RFP expression, which points to a low activity of the meis 1 minimal promoter. Lines ED48-ED272 were generated using the IIC construct, which was built as depicted in Figure 1, using an irx $3 a$ minimal promoter (Bessa et al. 2009) and mCherry as reporter gene in the RFP enhancer trap. The sequence of IIC is available in the Supplemental Material and on http://www. upo.es/CABD/EDscreen/ ("Methods and Protocols" section).

\section{Transgenesis and genetic screening}

The ED vector was injected in one-cell-stage zebrafish embryos (TU strain) together with Tol2 mRNA. The Tol2 transposon/transposase method of transgenesis (Kawakami et al. 2004) was used as previously described (Bessa et al. 2009). Injected embryos showing broad GFP or RFP expression at 24-48 hpf were selected and grown to adults. Stable transgenic lines were detected by outcrossing ED-injected fish with wild-type animals and isolated from these crosses showing in vivo GFP or RFP expression. Embryos were kept in E3 medium with 0.003\% 1-phenyl-2-thiourea (Sigma-Aldrich) to prevent pigmentation. Embryos showing GFP or RFP expression were anesthetized by adding tricaine (ethyl 3-aminobenzoate; Sigma-Aldrich) to the E3 medium, and expression patterns were documented at 24-36 hpf and at 48-60 hpf and annotated (Supplemental Table 1). Approximately half of the identified stable transgenic lines were selected to grow and were maintained by outcrossing with wild-type animals.

Mouse transgenesis was performed as previously reported (Sumiyama et al. 2010). The injected embryos were isolated at 10.5-11.5 d post-coitum (dpc) and documented for GFP or RFP expression. Genomic DNA was extracted from individually isolated extra-embryonic membranes and genotyped by PCR to identify those carrying the transposon. Fourteen out of 94 injected embryos were found to carry the ED transposon.

\section{Identification of ED integration sites}

ED integration sites were identified by inverse PCR. For each line, genomic DNA was extracted from five embryos separately, digested with Sau3AI (Roche), and then ligated with a T4 ligase (Promega). The ligated DNA was used as template for the inverse PCR. Nested PCR was performed for the two ends of the transposon. A list of 

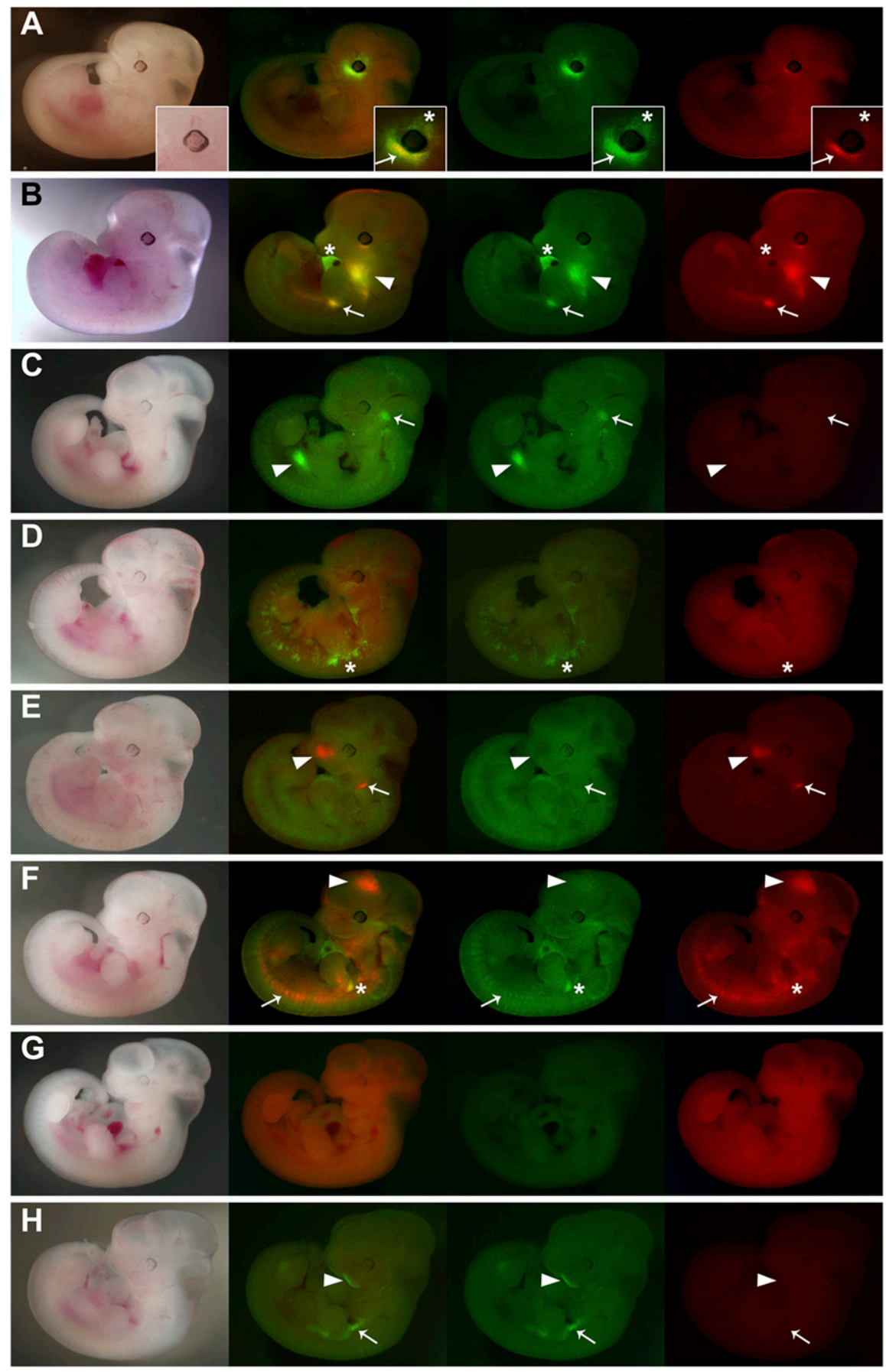

Figure 6. Mouse transgenic ED lines. Eight mouse transgenic embryos were generated using the ED system. ( $A$ ) In this example, GFP expression is detected in the dorsal periocular mesenchyme (asterisk; inset), and coexpression of GFP and RFP is observed in the ventral periocular mesenchyme (arrow; inset). (B) Strong GFP expression is detected in the heart (asterisk), while GFP and RFP coexpression is detected at the base of the forelimb (arrow), the branchial arches (arrowhead), and at low levels in the somites. (C) GFP expression is detected in the trigeminal ganglion (arrow) and the midgut (arrowhead), while RFP expression is not detected. (D) This embryo expresses GFP throughout the embryo in migratory cells that probably correspond to dermis (asterisk), while RFP is found ubiquitously at low levels. ( $E$ ) RFP expression is detected in the olfactory pits (arrowhead) and the second branchial arch (arrow), possibly in the mesodermal core, while GFP is detected at low levels ubiquitously. ( $F$ ) RFP expression alone is detected in the forebrain (arrowhead), somites (arrow), and cells migrating into the limb buds; RFP and GFP coexpression is strongly detected in the proximal region of the forelimb (asterisk), while unique GFP expression is detected at low levels ubiquitously. (G) GFP and RFP are coexpressed ubiquitously at low levels. (H) Expression of GFP alone is observed superficially in the mandibular component of the first branchial arch (arrowhead), in tissues surrounding the fore and hindlimbs (arrow), and RFP expression is not detected. The stages of the embryos shown are $11.5 \mathrm{~d}$ post-coitum (dpc; $A, B)$ and $10.5 \mathrm{dpc}(\mathrm{C}-\mathrm{H})$. The first column presents transmitted light images, the second an overlay of GFP and RFP channels, the third the GFP channel alone (green), and the fourth the RFP channel alone (red).

\section{Genome Research}


primers and PCR protocols for inverse PCR is available in Supplemental Table 3 . For the GFP end, 5TolF1 and 5TolR1 primers were used for the first round of PCRs, and 5TolF2 and 5TolR2 were used for the second round. For the RFP end, 3TolF1 and 3TolR1 primers were used for the first round of PCRs, and 3TolF2 and 3TolR2 or 3TolF2 and 3TolTest were used for the second round. The products of the second round of PCR were then visualized in a $2 \%$ agarose gel, and for each end, the common band for the five embryos was cloned in TOPO (Invitrogen, pCR8/GW/TOPO TA cloning KIT). TOPO clones were sequenced using a GW1 primer. Obtained sequences were analyzed to identify fragments adjacent to the transposon, which were then aligned to the zebrafish genome (Zv9/ danRer7 assembly using the UCSC Genome Browser BLAT web page; http://genome.ucsc.edu/cgi-bin/hgBlat). Integration sites were confirmed either by having the $5^{\prime}$ and $3^{\prime}$ hits in the same genomic position or by finding nearby genes with similar expression patterns to those observed with the reporter genes in the corresponding line. All sequences were long enough to map the transposon to a unique position, with the exception of ED98, for which two positions were annotated (Supplemental Table 2). Expression patterns of genes near integration points were analyzed, and if shared with the corresponding ED line, a gene-to-ED line association was established. Gene expression patterns were assessed initially using public available data (Bradford et al. 2011) (ZFIN; http://zfin.org/) and confirmed later by in situ hybridization. When no initial candidate gene was detected, in situ hybridization was performed for one to six different genes near the integration site.

\section{Southern blot}

One microgram of genomic DNA was extracted from adult animals and digested with PstI or EcoRV. The samples were then electrophoresed through 1\% agarose and Southern blotted using a digoxigenin (DIG)-labeled probe against GFP. Blots were revealed using CSPD and imaged in a gel imaging system (ChemiDoc XRS+, Bio-Rad).

\section{RNA probes and whole-mount in situ hybridization}

cDNAs were amplified by performing a PCR with gene-specific primers (primer sequences are available in Supplemental Table 3) using a mix of $80 \%$ epiboly to 48 -hpf retro-transcribed cDNA as template (SuperScript II, Invitrogen). After PCR amplification, specific cDNAs were cloned in a pGEM-T easy vector (Promega). These clones were linearized with restriction enzymes, and DIG-labeled RNA probes were synthesized using SP6 or T7 RNA polymerase (Roche), depending on the orientation of each insert. Wild-type embryos or ED transgenic embryos were used for whole-mount in situ hybridization. Embryos were fixed at 20-24 hpf in PFA (4\% in PBS) overnight, and whole-mount in situ hybridization was performed as reported (Tena et al. 2007). For in-crossed ED lines background, whole-mount in situ hybridizations were performed using a mix of two DIG-labeled RNA probes, the anti-sense of the putative ED-associated gene and the anti-sense of egr $2 b$, a gene expressed in rhombomeres 3 and 5 (Oxtoby and Jowett 1993) that we used as an internal in situ control. For the acquisition of fluorescent images, whole-mount in situ hybridizations developed with NBT/BCIP were stained overnight with the nuclear marker DAPI, confocal images were acquired using a Leica-SPE confocal system, and data were processed with Adobe Photoshop and ImageJ (Schneider et al. 2012).

\section{Cre recombinase injection}

A vector containing the Cre recombinase cDNA (Langenau et al. 2005) was linearized using the NotI restriction enzyme (Roche), and mRNA was synthesized using Sp6 RNA polymerase (Roche). One hundred to $200 \mathrm{pg}$ of mRNA was injected in one-cell-stage ED transgenic embryos. Embryos were grown to $20-24 \mathrm{hpf}$ at $28^{\circ} \mathrm{C}$, and injected and noninjected embryos were documented for GFP and RFP expression.

\section{Morpholino injections and confocal microscopy}

Two different amounts ( 3 or $10 \mathrm{ng}$ ) of a morpholino targeting ptrfb (5'- GACGGCTGTCTTCAATCACCTCCAT-3') (Hill et al. 2008) were injected in one- to two-cell-stage zebrafish embryos. Embryos were grown to $5 \mathrm{dpf}$ and fixed overnight at $4^{\circ} \mathrm{C}$ in $4 \%$ paraformaldehyde in PBS. Fixed embryos were stained with DAPI and Rhodamine Phalloidin, and analyzed in a Leica SPE confocal microscope.

\section{Quantitative PCR}

To see if ED186 insertion results in a decrease of transcription of mir124-5, quantitative PCR was used. Total mRNA was extracted from 48-hpf wild-type and ED186 homozygous embryos by TRIzol extraction (Invitrogen) and subsequent phenol:chloroform purification. Two independent biological replicas were used. 0.9-1.5 ng of total mRNA was used for reverse transcription with random primers (SuperScript II, Invitrogen). Quantitative PCR was performed on a Bio-Rad CFX96 real-time system using EvaGreen (SsoFast EvaGreen, Bio-Rad). Two rounds of qPCR were performed per biological replica, and triplicates were used per round of qPCR. Previously described primers for mir124-5 were used (Shkumatava et al. 2009), primers for eef1a1l1 were used as internal control, and expression levels were compared using the $\Delta \Delta C_{\mathrm{T}}$ method. The primers and PCR program are described in Supplemental Table 3.

\section{Chromosome conformation capture assays}

Twenty-four hpf wild-type and ED170 homozygous embryos were dissociated to single cells. Cells were fixed and lysed, and nuclei were then digested with HindIII endonuclease (Roche). HindIII was inactivated, and samples were treated with T4 DNA ligase (Promega). A set of locus-specific primers (Supplemental Table 3) was designed with the online program Primer3 v. 0.4.0 (Rozen and Skaletsky 2000) (http://frodo.wi.mit.edu/), each primer being close to a HindIII site flanking a chromosomal site of interest. Each DNA sample was calibrated so that the PCR products were always in the linear range. A primer next to the ptrfb promoter was considered fixed, and different interactions were tested using primers close to regions of interest, including enhancers and negative controls. Quantitative PCR was performed on a Bio-Rad CFX96 real-time system with SYBR Green (FastStart, Roche). PCR product values were related to a control composed of a bacterial artificial chromosome (BAC) that encompasses all of our regions of interest (CH73-162C2). To compare wild-type and ED170 samples, PCR values were normalized by means of control primers targeting the $X P B$ gene locus for which a BAC control was also used (DKEY-32H20). The primers and PCR program are described in Supplemental Table 3.

\section{Statistical analysis}

Assuming that the data are normally distributed, a one-tailed $t$-test was performed to test the significance of differences among sample averages. In all tests, we adopted an alpha level of 0.05 . Differences were considered significant or highly significant when $P$-values were situated below 0.05 or 0.01 , respectively.

\section{Acknowledgments}

This paper is dedicated to the memory of Robert Whittle, his inspiring spirit and his contributions to genetics. We thank Fernando 
Casares, Juan Ramón Martínez Morales, Marcelo Nobrega, Miguel Manzanares, and Ozren Bogdanovic for helpful suggestions and critical reading of the manuscript; and Juan Tena for 3C assistance. This study was supported by the Spanish and Andalusian Governments (JLGS grant numbers BFU2010-14839, CSD2007-00008, Proyecto de Excelencia CVI-3488, and JJC grant number BFU201122928), an EFSD/Lilly grant, and a Universidad Pablo de Olavide grant (JB grant number PPI0906). A.A.-C. is an FPI fellow and J.B. is a Juan de la Cierva postdoctoral fellow (JCI-2009-04014) of the Consejo Superior de Investigaciones Cientificas. J.B. was also an FCT postdoctoral fellow (SFRH/BPD/38829/2007; POPH/FSE). M.L. is a Junta de Andalucia fellow.

\section{References}

Bedell VM, Wang Y, Campbell JM, Poshusta TL, Starker CG, Krug Ii RG, Tan W, Penheiter SG, Ma AC, Leung AYH, et al. 2012. In vivo genome editing using a high-efficiency TALEN system. Nature 491: 114-118.

Bell AC, West AG, Felsenfeld G. 2001. Insulators and boundaries: Versatile regulatory elements in the eukaryotic genome. Science 291: 447-450.

Bessa J, Tena JJ, de la Calle-Mustienes E, Fernández-Miñán A, Naranjo S, Fernández A, Montoliu L, Akalin A, Lenhard B, Casares F, et al. 2009. Zebrafish enhancer detection (ZED) vector: A new tool to facilitate transgenesis and the functional analysis of cis-regulatory regions in zebrafish. Dev Dyn Off Publ Am Assoc Anat 238: 2409-2417.

Blow MJ, McCulley DJ, Li Z, Zhang T, Akiyama JA, Holt A, Plajzer-Frick I, Shoukry M, Wright C, Chen F, et al. 2010. ChIP-seq identification of weakly conserved heart enhancers. Nat Genet 42: 806-810.

Bogdanovic O, Fernandez-Miñán A, Tena JJ, de la Calle-Mustienes E, Hidalgo C, van Kruysbergen I, van Heeringen SJ, Veenstra GJC, Gómez-Skarmeta JL. 2012. Dynamics of enhancer chromatin signatures mark the transition from pluripotency to cell specification during embryogenesis. Genome Res 22: 2043-2053.

Bradford Y, Conlin T, Dunn N, Fashena D, Frazer K, Howe DG, Knight J, Mani P, Martin R, Moxon SAT, et al. 2011. ZFIN: Enhancements and updates to the Zebrafish Model Organism Database. Nucleic Acids Res 39: D822-D829.

Chang N, Sun C, Gao L, Zhu D, Xu X, Zhu X, Xiong J-W, Xi JJ. 2013. Genome editing with RNA-guided Cas9 nuclease in zebrafish embryos. Cell Res 23: 465-472.

Chung JH, Whiteley M, Felsenfeld G. 1993. A 5' element of the chicken $\beta$-globin domain serves as an insulator in human erythroid cells and protects against position effect in Drosophila. Cell 74: 505-514.

Collette NM, Genetos DC, Economides AN, Xie L, Shahnazari M, Yao W, Lane NE, Harland RM, Loots GG. 2012. Targeted deletion of Sost distal enhancer increases bone formation and bone mass. Proc Natl Acad Sci 109: 14092-14097.

Dekker J, Rippe K, Dekker M, Kleckner N. 2002. Capturing chromosome conformation. Science 295: 1306-1311.

Dixon JR, Selvaraj S, Yue F, Kim A, Li Y, Shen Y, Hu M, Liu JS, Ren B. 2012. Topological domains in mammalian genomes identified by analysis of chromatin interactions. Nature 485: 376-380.

Doyon Y, McCammon JM, Miller JC, Faraji F, Ngo C, Katibah GE, Amora R, Hocking TD, Zhang L, Rebar EJ, et al. 2008. Heritable targeted gene disruption in zebrafish using designed zinc-finger nucleases. Nat Biotechnol 26: 702-708.

Driever W, Solnica-Krezel L, Schier AF, Neuhauss SC, Malicki J, Stemple DL, Stainier DY, Zwartkruis F, Abdelilah S, Rangini Z, et al. 1996. A genetic screen for mutations affecting embryogenesis in zebrafish. Development 123: $37-46$.

The ENCODE Project Consortium. 2012. An integrated encyclopedia of DNA elements in the human genome. Nature 489: $57-74$.

Epstein DJ. 2009. Cis-regulatory mutations in human disease. Brief Funct Genomics Proteomics 8: $310-316$.

Gardiner MR, Gongora MM, Grimmond SM, Perkins AC. 2007. A global role for zebrafish klf4 in embryonic erythropoiesis. Mech Dev 124: 762-774.

Geyer PK, Corces VG. 1992. DNA position-specific repression of transcription by a Drosophila zinc finger protein. Genes Dev 6: 1865 1873.

Ghiasvand NM, Rudolph DD, Mashayekhi M, Brzezinski JA IV, Goldman D, Glaser T. 2011. Deletion of a remote enhancer near ATOH7 disrupts retinal neurogenesis, causing NCRNA disease. Nat Neurosci 14: 578-586.

Giraldo P, Martínez A, Regales L, Lavado A, García-Díaz A, Alonso A, Busturia A, Montoliu L. 2003. Functional dissection of the mouse tyrosinase locus control region identifies a new putative boundary activity. Nucleic Acids Res 31: 6290-6305.
Golling G, Amsterdam A, Sun Z, Antonelli M, Maldonado E, Chen W, Burgess S, Haldi M, Artzt K, Farrington S, et al. 2002. Insertional mutagenesis in zebrafish rapidly identifies genes essential for early vertebrate development. Nat Genet 31: 135-140.

Haffter P, Granato M, Brand M, Mullins MC, Hammerschmidt M, Kane DA, Odenthal J, van Eeden FJ, Jiang YJ, Heisenberg CP, et al. 1996. The identification of genes with unique and essential functions in the development of the zebrafish, Danio rerio. Development 123: 1-36.

Hill MM, Bastiani M, Luetterforst R, Kirkham M, Kirkham A, Nixon SJ, Walser P, Abankwa D, Oorschot VMJ, Martin S, et al. 2008. PTRF-Cavin, a conserved cytoplasmic protein required for caveola formation and function. Cell 132: 113-124.

Hwang WY, Fu Y, Reyon D, Maeder ML, Tsai SQ, Sander JD, Peterson RT, Yeh J-RJ, Joung JK. 2013. Efficient genome editing in zebrafish using a CRISPR-Cas system. Nat Biotechnol 31: 227-229.

Jeong Y, Leskow FC, El-Jaick K, Roessler E, Muenke M, Yocum A, Dubourg C, Li X, Geng X, Oliver G, et al. 2008. Regulation of a remote Shh forebrain enhancer by the Six3 homeoprotein. Nat Genet 40: 1348-1353.

Kawakami K, Shima A, Kawakami N. 2000. Identification of a functional transposase of the Tol2 element, an Ac-like element from the Japanese medaka fish, and its transposition in the zebrafish germ lineage. Proc Natl Acad Sci 97: 11403-11408.

Kawakami K, Takeda H, Kawakami N, Kobayashi M, Matsuda N, Mishina M. 2004. A transposon-mediated gene trap approach identifies developmentally regulated genes in zebrafish. Dev Cell 7: 133-144.

Kilpinen H, Dermitzakis ET. 2012. Genetic and epigenetic contribution to complex traits. Hum Mol Genet 21: R24-R28.

Kirkland JG, Raab JR, Kamakaka RT. 2013. TFIIIC bound DNA elements in nuclear organization and insulation. Biochim Biophys Acta 1829: 418424.

Kleinjan D-J, Coutinho P. 2009. Cis-ruption mechanisms: Disruption of cisregulatory control as a cause of human genetic disease. Brief Funct Genomics Proteomics 8: $317-332$.

Kwan KM, Fujimoto E, Grabher C, Mangum BD, Hardy ME, Campbell DS, Parant JM, Yost HJ, Kanki JP, Chien C-B. 2007. The Tol2kit: A multisite gateway-based construction kit for Tol2 transposon transgenesis constructs. Dev Dyn Off Publ Am Assoc Anat 236: 3088-3099.

Langenau DM, Feng H, Berghmans S, Kanki JP, Kutok JL, Look AT. 2005. Cre/ lox-regulated transgenic zebrafish model with conditional $m y c$-induced T cell acute lymphoblastic leukemia. Proc Natl Acad Sci 102: 6068-6073.

Lettice LA, Horikoshi T, Heaney SJH, van Baren MJ, van der Linde HC, Breedveld GJ, Joosse M, Akarsu N, Oostra BA, Endo N, et al. 2002. Disruption of a long-range cis-acting regulator for Shh causes preaxial polydactyly. Proc Natl Acad Sci 99: 7548-7553.

Martin D, Pantoja C, Fernández Miñán A, Valdes-Quezada C, Moltó E, Matesanz F, Bogdanović O, de la Calle-Mustienes E, Domínguez O, Taher $\mathrm{L}$, et al. 2011. Genome-wide CTCF distribution in vertebrates defines equivalent sites that aid the identification of disease-associated genes. Nat Struct Mol Biol 18: 708-714.

Maurano MT, Humbert R, Rynes E, Thurman RE, Haugen E, Wang H, Reynolds AP, Sandstrom R, Qu H, Brody J, et al. 2012. Systematic localization of common disease-associated variation in regulatory DNA. Science 337: 1190-1195.

Meng X, Noyes MB, Zhu LJ, Lawson ND, Wolfe SA. 2008. Targeted gene inactivation in zebrafish using engineered zinc-finger nucleases. Nat Biotechnol 26: 695-701.

The Mouse ENCODE Consortium. 2012. An encyclopedia of mouse DNA elements (Mouse ENCODE). Genome Biol 13: 418.

Oxtoby E, Jowett T. 1993. Cloning of the zebrafish krox-20 gene $(k r x-20)$ and its expression during hindbrain development. Nucleic Acids Res 21: 1087-1095.

Phillips-Cremins JE, Corces VG. 2013. Chromatin insulators: Linking genome organization to cellular function. Mol Cell 50: 461-474.

Ragvin A, Moro E, Fredman D, Navratilova P, Drivenes Ø, Engström PG, Alonso ME, de la Calle Mustienes E, Gómez Skarmeta JL, Tavares MJ, et al. 2010. Long-range gene regulation links genomic type 2 diabetes and obesity risk regions to HHEX, SOX4, and IRX3. Proc Natl Acad Sci 107: 775-780.

Recillas-Targa F, Pikaart MJ, Burgess-Beusse B, Bell AC, Litt MD, West AG, Gaszner M, Felsenfeld G. 2002. Position-effect protection and enhancer blocking by the chicken $\beta$-globin insulator are separable activities. Proc Natl Acad Sci 99: 6883-6888.

Rozen S, Skaletsky H. 2000. Primer3 on the WWW for general users and for biologist programmers. Methods Mol Biol 132: 365-386.

Ruf S, Symmons O, Uslu VV, Dolle D, Hot C, Ettwiller L, Spitz F. 2011. Largescale analysis of the regulatory architecture of the mouse genome with a transposon-associated sensor. Nat Genet 43: 379-386.

Sanyal A, Lajoie BR, Jain G, Dekker J. 2012. The long-range interaction landscape of gene promoters. Nature 489: 109-113.

Schneider CA, Rasband WS, Eliceiri KW. 2012. NIH Image to ImageJ: 25 years of image analysis. Nat Methods 9: 671-675.

\section{Genome Research}


Shkumatava A, Stark A, Sive H, Bartel DP. 2009. Coherent but overlapping expression of microRNAs and their targets during vertebrate development. Genes Dev 23: 466-481.

Spitz F, Gonzalez F, Duboule D. 2003. A global control region defines a chromosomal regulatory landscape containing the HoxD cluster. Cell 113: 405-417.

Sumiyama K, Kawakami K, Yagita K. 2010. A simple and highly efficient transgenesis method in mice with the Tol2 transposon system and cytoplasmic microinjection. Genomics 95: 306-311.

Tena JJ, Neto A, de la Calle-Mustienes E, Bras-Pereira C, Casares F, GómezSkarmeta JL. 2007. Odd-skipped genes encode repressors that control kidney development. Dev Biol 301: 518-531.

Urasaki A, Morvan G, Kawakami K. 2006. Functional dissection of the Tol2 transposable element identified the minimal cis-sequence and a highly repetitive sequence in the subterminal region essential for transposition. Genetics 174: 639-649.
Visel A, Blow MJ, Li Z, Zhang T, Akiyama JA, Holt A, Plajzer-Frick I, Shoukry M, Wright C, Chen F, et al. 2009. ChIP-seq accurately predicts tissuespecific activity of enhancers. Nature 457: 854-858.

Visel A, Zhu Y, May D, Afzal V, Gong E, Attanasio C, Blow MJ, Cohen JC, Rubin EM, Pennacchio LA. 2010. Targeted deletion of the 9p21 noncoding coronary artery disease risk interval in mice. Nature 464: 409-412.

Wienholds E, van Eeden F, Kosters M, Mudde J, Plasterk RHA, Cuppen E. 2003. Efficient target-selected mutagenesis in zebrafish. Genome Res 13: $2700-2707$.

Wienholds E, Kloosterman WP, Miska E, Alvarez-Saavedra E, Berezikov E, de Bruijn E, Horvitz HR, Kauppinen S, Plasterk RHA. 2005. MicroRNA expression in zebrafish embryonic development. Science 309: 310-311.

Received August 26, 2013; accepted in revised form November 20, 2013. 


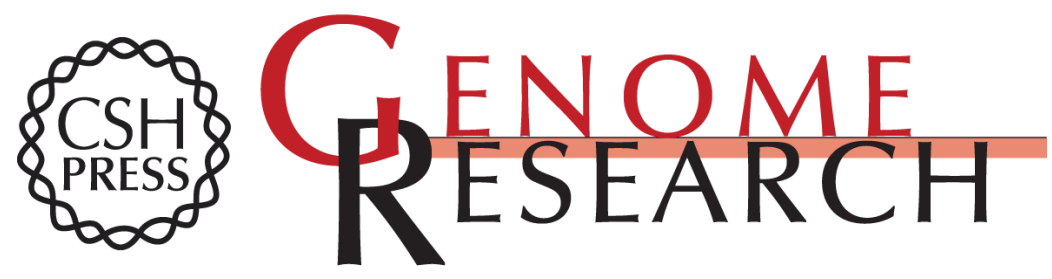

\section{A mobile insulator system to detect and disrupt cis-regulatory landscapes in vertebrates}

José Bessa, Mario Luengo, Solangel Rivero-Gil, et al.

Genome Res. 2014 24: 487-495 originally published online November 25, 2013

Access the most recent version at doi:10.1101/gr.165654.113

\section{Supplemental} Material

References

Creative

Commons

License

Email Alerting Service
http://genome.cshlp.org/content/suppl/2014/01/06/gr.165654.113.DC1

This article cites 53 articles, 17 of which can be accessed free at: http://genome.cshlp.org/content/24/3/487.full.html\#ref-list-1

This article is distributed exclusively by Cold Spring Harbor Laboratory Press for the first six months after the full-issue publication date (see

http://genome.cshlp.org/site/misc/terms.xhtml). After six months, it is available under a Creative Commons License (Attribution-NonCommercial 3.0 Unported), as described at http://creativecommons.org/licenses/by-nc/3.0/.

Receive free email alerts when new articles cite this article - sign up in the box at the top right corner of the article or click here.

\section{Affordable, Accurate Sequencing.}

To subscribe to Genome Research go to:

https://genome.cshlp.org/subscriptions 